\title{
Study on Power Saving Based on Radio Frame in LTE Wireless Communication System Using DRX
}

\author{
Scott Fowler
}

\section{Linköping University Post Print}

N.B.: When citing this work, cite the original article.

C2011 IEEE. Personal use of this material is permitted. However, permission to reprint/republish this material for advertising or promotional purposes or for creating new collective works for resale or redistribution to servers or lists, or to reuse any copyrighted component of this work in other works must be obtained from the IEEE.

Scott Fowler , Study on Power Saving Based on Radio Frame in LTE Wireless Communication System Using DRX, 2011, IEEE Globecom Workshop on Workshop of SCPA and SaCoNAS. 


\title{
Study on Power Saving Based on Radio Frame in LTE Wireless Communication System Using DRX
}

\author{
Scott Fowler \\ Linköping University, Department of Science and Technology \\ SE-601 74, Norrköping, Sweden
}

\begin{abstract}
Long Term Evolution (LTE) of 3GPP provides high bandwidth for data transfer. Having high bandwidth transfer will exhaust the user equipment (UE)'s battery power quickly. To extend the UE's battery lifetime the 3GPP standards for LTE support Enhanced Discontinuous Reception (DRX). The DRX allow an idle UE to save battery by turning off the radio receiver for a predefined period. Since the Transmission Time Interval (TTI) is related to the size of the data block passed from the higher networks layers to the radio link layer, the DRX scheduler should vary the resource allocation of each user basis on TTI. In this paper, we have evaluated the influences of the TTI sizes as well as the effects of LTE DRX Light and Deep Sleep mode on power consumption for Voice and Web traffic.
\end{abstract}

\section{INTRODUCTION}

Wireless World Research Forum (WWRF) forecasted that 7 trillion wireless devices would serve 7 billion users by 2017 [1]. This is evident in the explosion of powerful mobile devices in the market; introduction of the smartphones (e.g. iPhone) and the new tablets (e.g. iPad) as well as the continuing growth of netbooks and laptops. These devices are capable of supporting new services (e.g. Skype, GTalk, Twitter) for web, video and voice traffic. As a result, wireless devices need to be powerful enough to deliverer a wide range of traffic. However, due to the low transmission rate and high service costs, the $3 \mathrm{G}$ (third generation) technology has been unsuccessful in delivering high-speed mobile broadband.

To address the mobile broadband requirements, the International Telecommunication Unions (ITU) launched the International Mobile Telecommunications (IMT)-Advanced initiative in order to develop fourth generation network (4G) technologies. One of the technologies is Long-Term Evolution (LTE), that is being developed by the Third Generation Partnership Project (3GPP; www.3gpp.org). LTE is designed to be a high data rate and low latency system aiming to support different types of services including web browsing, FTP, video streaming, VoIP, online gaming and real time video.

There has been investigation on the short Transmission Time Interval (TTI) of $1 \mathrm{~ms}$ by means of TTI bundling [2]-[4]. The TTI bundling allows repeating the same data in multiple TTIs, which effectively increases the TTI length, thus allowing the UE to transmit for a longer time. The advantage over sending the packet in a single TTI, compared to a several small TTI (example $1 \mathrm{~ms}$ ) and not received correctly, will result in having one or more retransmissions. This retransmission results in a lot of signalling overhead since small TTI requires more retransmission. Latency is also reduced, since no waiting time is required between the retransmissions in a single TTI.
With the use of the enriched $4 \mathrm{G}$ services, it is still currently limited because the receivers for these services require computationally complex circuitry that drains the user equipment (UE)s battery power quickly. Hence, one of the main objectives of LTE is to provide very efficient power conservation mechanisms to achieve longer battery life. One prominent option to prolong the battery life is the use of discontinuous reception (DRX) [5]-[8] in LTE. The DRX was introduced by 3GPP in [9], which allows an idle UE to save battery by turning off the radio receiver for a predefined period. This allows for significant improvement in resource utilization, particularly for applications. However, there has been no consideration how the TTI effects the DRX in LTE in order to optimize the DRX parameters, so as to maximize power saving, therefore the objective of this paper is to compare the influences of TTI sizes as well as the effects of DRX Light and Deep Sleep mode for Voice and Web traffic.

\section{RELATED WORK}

Various methods have studied power saving [6]-[8], [11][13]. In [11], an adjustable power management using a hierarchical cascaded power gating and a hierarchical multilevel clock gating was proposed. To have the maximum power saving in the LTE five power saving classes with a combination of power gating were defined. However this work did not consider bursty traffic such as voice. In [12] a DRX-aware scheduling (DAS) method is proposed for video services in wireless downlink systems. The idea is that, a UE which is going soon to return to sleep should have priority. However, there was no analysis of different active time in the form of power function with different users. In-depth analysis to explore the optimal choice of power function should be carefully studied.

Other papers have studied DRX for power saving. In [8], a variant of the M/G/1 queues was presented in order to explore the performance of the DRX. The closed-form equations are derived for the output measures based on the Poisson assumption, and a novel semi-Markov process is proposed to model the DRX in [7]. With [6] provided a comparison of the energy saving mechanisms of 3GPP and 3GPP2. The work in [13] presented an overview of an adjustable feature of the DRX Cycle of the LTE DRX using a semi-Markov process. These methods did not consider various packet arrival patterns and scheduling behaviors of system in relation to power efficiency.

All these works did not consider how TTI Radio Frame 
plays an important role in relation to DRX for power consumption.

\section{LTE AND THE DRX CONCEPT}

LTEs power efficient strategy exploits the concepts of DRX and Discontinuous Transmission (DTX) [14]. By using DRX, the terminal can turn the radio frequency modem into sleep mode for prolonged period either in order to extend battery life.

In LTE DRX mechanism, the sleep/wake scheduling of each UE is determined by the following four parameters: DRX Short Cycle $\left(t_{D S}\right)$, DRX Long Cycle $\left(t_{D L}\right)$, DRX Inactivity Timer $\left(t_{I}\right)$ and DRX Short Cycle Timer $\left(t_{N}\right)$. The $t_{D S}$ and $t_{D L}$ define duration of ON period, which is fixed value applied to both Long and Short Cycles ${ }^{1}$. The UE monitors the physical downlink control channel (PDCCH) to determine if there is any transmission over the shared data channel allocated to the UE during the ON duration.

In LTE DRX, the sleep/wake-up mode consists of the three different states, namely, Inactivity period, Light Sleep period, and Deep Sleep period. The Inactivity period is the power active mode, whereas the Light Sleep period and the Deep Sleep period are the power saving mode. The transition from the Inactivity period to the Light Sleep period is controlled by $t_{I}$, while the transition from the Light Sleep period to the Deep Sleep period within the power saving mode is controlled by $t_{N}$.

The following describes how the UE receiver works during the Inactivity period, Light Sleep period, and Deep Sleep period [10]. Note that this study focuses on the DRX, where the DRX Cycles are adjustable and not fixed. Here, we will assume that the UE does not go to sleep during the time between first transmission of the packet and the retransmission of the same packet.

During the Inactive period, DRX Inactivity Timer is running and the UE receiver is also turned ON. In this period the UE receiver monitors the $\mathrm{PDCCH}$ and receives packets delivered via eNB from the Evolved Packet Core. If a downlink transmission is indicated by the PDCCH before the DRX Inactivity Timer is expired, the next DRX Inactivity Timer kicks in. If no downlink transmission is indicated, then the Inactivity period is terminated with the expiration of DRX Inactivity Timer.

During the Light Sleep period, DRX Short Cycle Timer is active. To monitor the PDCCH, the UE wakes up before the DRX Short Cycle Timer expires, which is the ON duration of each DRX Short Cycle. If a downlink transmission is indicated by the PDCCH, the UE wakes up and the DRX Inactivity Timer kicks in. This is the transition from the Light Sleep period to the Inactivity period which is the power active mode. If no transmission is indicated, the UE stays sleeping until the next ON duration.

During the Deep Sleep period, the UE receiver status transition follows DRX Long Cycle. The UE wakes up and

\footnotetext{
${ }^{1}$ In UMTS there is no DRX Short Cycle or DRX Long Cycle, just fixed length DRX Cycles.
}

monitor the PDCCH during the ON duration of DRX Long Cycle. If a downlink transmission is indicated by the PDCCH three things will happen; UE Activates Timer, DRX Inactivity kicks in, and Light Sleep period transits to the Inactivity period. When no transmission is indicated, then the UE goes to sleep until the next $\mathrm{ON}$ duration. There will be no packet transition from the eNB processor to the UE during the Deep Sleep period either.

Essentially, capacity of PDCCH in the downlink ${ }^{2}$ depends on the number of multiplexed users per TTI for scheduling radio frame resources. Since the availability of $\mathrm{PDCCH}$ resources is limited, the amount of multiplexed users per TTI is also restricted when TTI is fixed. Contrarily, by varying the TTI dynamically ( i.e. increasing TTI when it is available) one may achieve the increased amount of multiplexed users per TTI. Longer TTI means increased amount of the data transmitted without increasing power consumption.

Thus, the TTI parameter in the layer should be configured so as to meet the performance requirement with minimize power consumption. A TTI with different size in the DRX Light and Deep Sleep mode could enhance the UE power consumption selected such that the power and resource savings are maximized. Investigating how Voice traffic and Web traffic will help in understanding the way the system has to consume more power to achieve a desired link performance in a channel.

\section{System Model}

To understand the power usage, the model is tested with two types of traffic with different characteristics; Voice and Web. Voice traffic can be modeled well by the Poisson process with exponential inter-arrival times because there are three possible situations in Voice traffic; both of the participants are silent, exactly one of them is speaking, or both are speaking at the same time. Unlike Voice traffic, Web traffic exhibits burstiness and correlations across an extremely wide range of time scales [15], [16].

\section{A. VoIP Traffic}

In this study, we applied a 4-state model for generating artificial conversational speech proposed in [17]. The Voice traffic model is based on conversational speech of three different languages, namely English, Italian and Japanese. Talk-spurts and pauses are generated according to the state transition model depicted in the Figure 1 . In the model, $\mathrm{P}_{1}, \mathrm{P}_{2}$, and $\mathrm{P}_{3}$ denote transition probabilities expressed ${ }^{3}$ in percentages. Two speakers, A and B, are engaged in a conversation, and the four states are as follows: State 1) A is talking and B is silent; State 2) mutual silence; State 3) A is silent and B is talking; State 4) double-talking.

ST (single talk duration), DT (double talk duration), and MS (mutual silence duration) vary according to the following equations. The times in these equations are expressed in seconds: $S T=-0.854 \ln (1-x 1) ; D T=-0.226 \ln (1-x 2)$;

\footnotetext{
${ }^{2}$ In the uplink, the resolution of the Channel Quality Indicator (CQI) depends on the capacity of Physical Uplink Control Channel (PUCCH).

${ }^{3}$ The values of $\mathrm{P}_{1}, \mathrm{P}_{2}$, and $\mathrm{P}_{3}$ are 40,50 , and 50, respectively.
} 


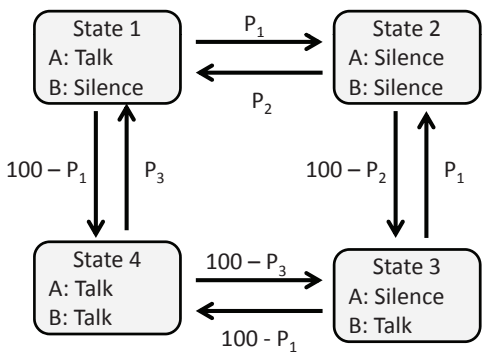

Fig. 1: State Transition Model for Conversation.

$M S=-0.854 \ln (1-x 3): 0<x 1, x 2, x 3>1$, random variable with uniform distribution.

\section{B. Web Traffic}

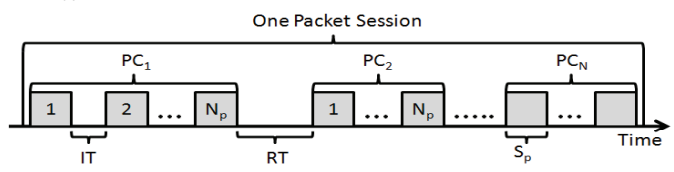

Fig. 2: Web Traffic Model.

The Web traffic model applied in this study is depicted in Figure 2. The user creates a session, consisting of a random number of packet calls (PCs), where each PC has a random number of packets, and duration or length [18].

The statistical distribution of the Web source model follows the recommendation in [18] and is summarized as follows.

- The geometric distribution is a discrete version of the exponential distribution.

- The exponential distribution is used to replace the geometric distribution for continuous random variables.

- The exponential distribution is a generalized Pareto distribution (GPD), given by:

$$
G P D(x ; \alpha ; k)= \begin{cases}1-\left(1-\frac{k x}{\alpha}\right)^{\frac{\alpha}{k}}, & k \neq 0 \\ 1-e^{-\frac{x}{k}}, & k=0\end{cases}
$$

where $k$ is the shape parameter and $\alpha>0$ is the scale parameter. For $k=0$, GPD reduces to an exponential distribution with mean $\alpha$, while for $k<0$ it is reduced to standard Pareto distribution.

Pareto with cutoff is used in the Web traffic model, where the packet size is defined with the following formula: PacketSize $=\min (P, m)$, where $P$ is normal Pareto distributed random variable ( $\alpha=1.1, k=81.5$ bytes) and $m$ is maximum allowed packet size (typically, $m=66666$ bytes). The PDF of the Packet Size is the probability that $\mathrm{x}>m$, by calculating as:

$f\left(\mu_{n}\right)=\frac{\alpha k-m\left(\frac{k}{m}\right)^{\alpha}}{\alpha-1}, \quad \alpha>1 \quad$ and $\quad k \leq x>m$

With the parameters above the average size is $f\left(\mu_{n}\right)=480$ bytes. Table I summarizes the characteristics of Web traffic model parameters.

TABLE I: Table Web Traffic Parameters

\begin{tabular}{|l|l|l|l|}
\hline \multicolumn{4}{|c|}{ TABLE } \\
\hline Definitions & Description & Distribution & Values \\
\hline $\mathrm{PC}_{n}$ & Number of PCs in a given session & Geometric & 5 \\
\hline $\mathrm{RT}$ & Reading time between PCs & Geometric & 412 seconds \\
\hline $\mathrm{N}_{P}$ & Number of packets within a PC & Geometric & 25 \\
\hline $\mathrm{IT}$ & Packet inter-arrival time & Geometric & Bit-rate Dependent \\
\hline $\mathrm{S}_{P}$ & Packet size or length & Pareto (with cutoff) & $\alpha=1.1, \mathrm{k}=81.5$ bytes \\
\hline
\end{tabular}

\section{UE Power Consumption Model}

The UE battery saving opportunities is calculated according to the model presented in 3GPP contribution [19] as shown in Figure 3. Three different states of UE activity are selected; Active, Light Sleep and Deep Sleep. Between each of the states a fixed transition time is applied, during which there is certain level of power consumption. The Power consumption levels are collected together and the length of the UE TTIs in order to calculate an overall estimate of the UE power consumption.

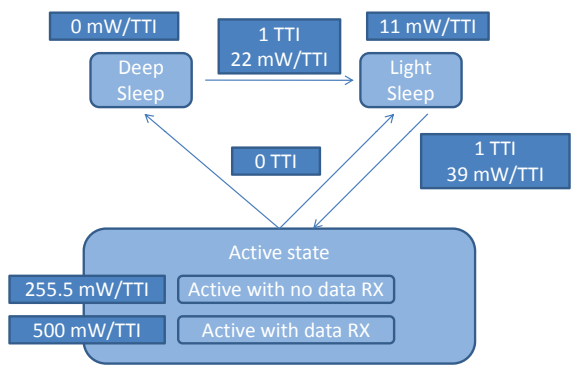

Fig. 3: UE Power Consumption Model.

\section{Results}

We have measured power usage for Voice and Web traffic in three different scenarios; Active, Light Sleep and Deep Sleep. Active denotes that regardless of the presence or absence of data, the UE stayed only in the active state, and there is no transition to the Deep nor Light Sleep mode. Light Sleep denotes, that when there is no data stream, the mode switches from Active state to Light Sleep state. Deep Sleep denotes, that, when there is no data stream, the mode switches from the Active state to the Deep Sleep state.

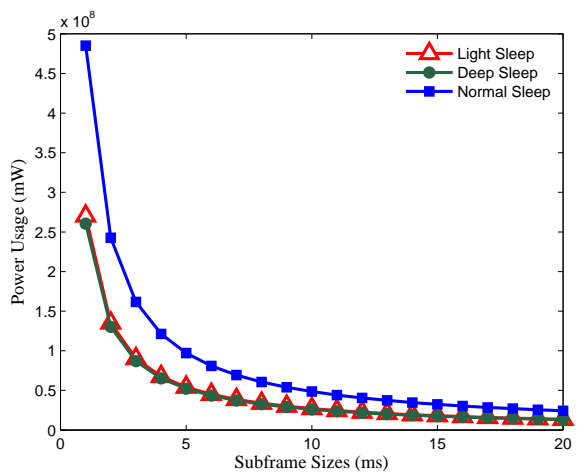

Fig. 4: Power Usage for TTI on Voice Traffic.

\section{A. Voice Traffic}

Figure 4 shows how much power is used when TTI are Static. The $\mathrm{x}$-axis indicates TTI subframe size in $\mathrm{ms}$, while $\mathrm{y}-$ axis shows power consumption of each state in $\mathrm{mW}$. The range of $1 \mathrm{~ms}$ to $20 \mathrm{~ms}$ was selected for the TTI. The Maximum TTI at $20 \mathrm{~ms}$ was used based on [18], where they found that Voice traffic typically has a $20 \mathrm{~ms}$ polling.

There is a general tendency in all three states that with an increase in the TTI subframe size, the power reduction occurs in an exponential decay manner. This trend is more noticeable in the Active state compared to Light or Deep Sleep mode. In 
the Active state, the power usage at subframe of $1 \mathrm{~ms}$ was 4.85 $\mathrm{X} 10^{8} \mathrm{~mW}$ then it was dropped to $2.42 \times 10^{8} \mathrm{~mW}$ at subframe size of $20 \mathrm{~ms}$, which was $2.42 \times 10^{7} \mathrm{~mW} / \mathrm{ms}$ reduction rate. On the other hand, in the Light Sleep state, the power usage at subframe of $1 \mathrm{~ms}$ was $2.70 \times 10^{8} \mathrm{~mW}$, and dropped to 1.35 $\mathrm{X} 10^{8} \mathrm{~mW}$ at subframe size of $20 \mathrm{~ms}$, which has a $1.35 \mathrm{X}$ $10^{7} \mathrm{~mW} / \mathrm{ms}$. Similarly in the Deep Sleep state, power usage reduction from subframe size $2.60 \times 10^{8} \mathrm{~mW}$ to $1.30 \times 10^{8}$ $\mathrm{mW}$ was $1.30 \times 10^{8} \mathrm{~mW} / \mathrm{ms}$ reduction rate. This shows Active state has a 2-fold reduction rate compared to Light or Deep Sleep modes.

Higher power consumption with small TTI may be explained by the fact that when each TTI is small it results in large number of subframes required in total. Thus by increasing TTI size, one can decrease the total subframe number thereby reducing power usage. More specifically, in Active state, there are two possible situations (Figure 4); Active with no data RX, which consumes $255.5 \mathrm{~mW} / \mathrm{TTI}$, or Active with data RX, which consumes $500 \mathrm{~mW} / \mathrm{TTI}$. When TTI size is large (e.g. $20 \mathrm{~ms}$ ) large amounts of data can be transmitted with just a single TTI subframe (e.g. <one active with data $>$ consumes $500 \mathrm{~mW} / \mathrm{TTI})$. The same amount of data may require more than one subframe when the size of TTI is small. In addition, with small TTI, there may be more < active without data $>$ subframes. Although power requirement is lower than $<$ active with data $>,<$ active without data $>$ can be seen as complete waste of the power. By widening the window of TTI, one can reduce the chance of $<$ active without data $>$ to occur.

In this scenario, Deep Sleep and Light Sleep appeared to use similar amount of power. It is due to the fact that the UE is in the power saving mode for only a small amount of time. The UE uses power saving mode when there is mutual silence. However, in real life telephone conversation, it is most likely that both parties start talking, or one of the parties start-talking, and there is little mutual silence.

\section{B. Dynamic TTI}

Unlike Static TTI, Dynamic TTI model consciously changes its size as the conversation changes states. In real life, conversation between two people will be in one of the four states; State 1) A is talking and B is silent; State 2) mutual silence; State 3) A is silent and B is talking; State 4) double-talking.

Our expectation is that the Dynamic model will maximize TTIs capacity by consciously adjusting its size based on the conversation states. In this study, we have set the range of adjustable TTI size between $10 \mathrm{~ms}$ and $20 \mathrm{~ms}$.

In the case of a mutual silence, the TTI will be set to the maximum $(20 \mathrm{~ms})$. In this state, the UE goes into the sleep mode (Light Sleep or Deep Sleep), and power consumption should be reduced. We have assessed the magnitude of power efficiency performance of Light Sleep and Deep Sleep mode.

If one person is talking while the other person is silent, the TTI will also be set to the maximum of $20 \mathrm{~ms}$. In the case of double talk (both parties talking), the TTI will be set to $10 \mathrm{~ms}$ for one party and the other party will have the rest of TTI (10 ms). The Dynamic TTI is enabled by the PDCCH. PDCCH informs the UE about the conversation status of the traffic in the downlink. The UE, then will be able to make decision on how to adjust the TTI accordingly.

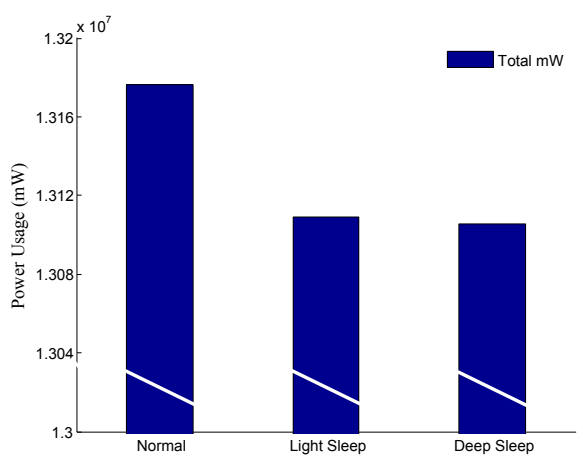

Fig. 5: Result of the adjustable TTI

Figure 5 shows the power usage of the Active, Light Sleep, and Deep Sleep tested on the Voice traffic with the adjustable TTI model. The y-axis represents actual total power usage during the testing period of 40 minutes in $\mathrm{mW}$.

Similarly to the Static TTI model, Deep Sleep and Light Sleep have comparable levels of power saving. Compared to Active, Deep Sleep uses approximately $70 \mathrm{~mW}$ less power and Light Sleep uses approximately $67 \mathrm{~mW}$ less power. Deep Sleep is slightly more power efficient than Light Sleep only by $4 \%$ relative to the Active state. Given the advantage of Light Sleep for minimum delay as discussed in the Static model, applying Light Sleep rather than Deep Sleep is the optimal power saving mode in the Dynamic Voice model as well.

\section{Web Traffic}

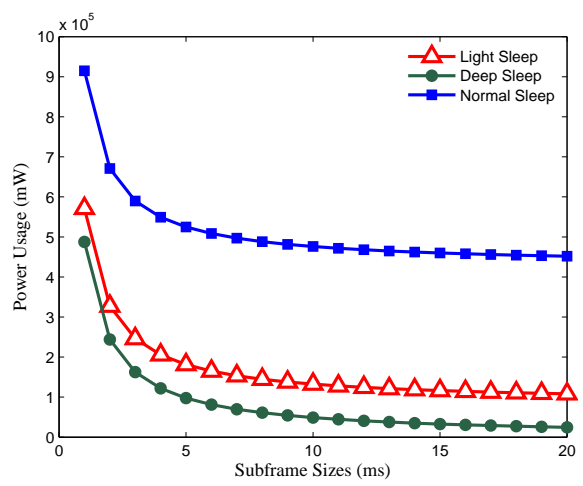

Fig. 6: Power Usage for TTI using Web Traffic

Figure 6 shows how much power is used in the Web traffic model. TTI was static considering the nature of the Web traffic. The $\mathrm{x}$-axis indicates TTI subframe size in $\mathrm{ms}$, while $\mathrm{y}$-axis shows power consumption of each state in $\mathrm{mW}$. The TTI range was between $1 \mathrm{~ms}$ and $20 \mathrm{~ms}$.

Similarly to the Voice traffic, there is a general tendency in all three states that as the TTI subframe size becomes larger, less power is required. This trend is more noticeable in Active state compared to Light or Deep Sleep modes. In Active state, the power usage at subframes of $1 \mathrm{~ms}$ was $9.15 \times 10^{5} \mathrm{~mW}$, then dropped to $4.52 \times 10^{5} \mathrm{~mW}$ at subframe sizes of $20 \mathrm{~ms}$ second, which was a $51 \%$ reduction. On the other hand, in the 
Light Sleep state, the power usage at subframe of $1 \mathrm{~ms}$ was $5.71 \times 10^{5} \mathrm{~mW}$, and dropped to $1.08 \times 10^{5} \mathrm{~mW}$ at subframe size of $20 \mathrm{~ms}$, which was only $80 \%$ reduction. Similarly in the Deep Sleep state, power usage reduction from subframe size $1 \mathrm{~ms}$ to $20 \mathrm{~ms}$ was $95 \%$.

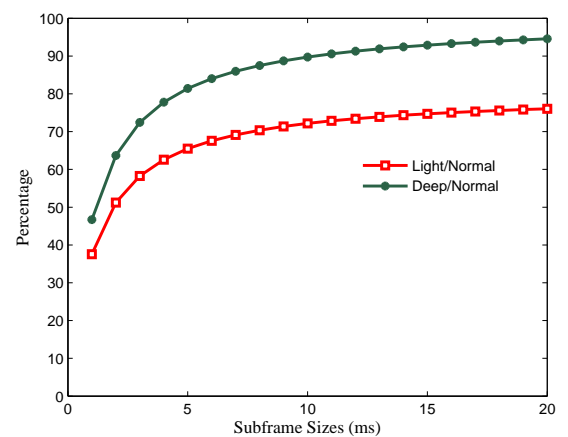

Fig. 7: \% usage in relation to Normal Power for Web Traffic

In order to closely compare the power efficiency of Light Sleep and Deep Sleep in Web traffic, the power usage was expressed in percentage difference relative to the Active State at each frame size (Figure 7). The $\mathrm{x}$-axis represents frame sizes in $\mathrm{ms}$, whereas $\mathrm{y}$-axis indicates percentage power usage: (Light Sleep- Active State) /Active State or (Deep SleepActive State)/Active State.

Both Light and Deep Sleep improve power efficiency as the frame size increase. However, Deep Sleep clearly demonstrated better power efficiency performance compared to Light Sleep, unlike the case of Voice traffic. Average power usage from frame size of 1 to $20 \mathrm{~ms}$ in Active State, Light Sleep, and Deep Sleep was $515 \mathrm{~mW}, 171 \mathrm{~mW}$ and $87 \mathrm{~mW}$, respectively. In other words, Deep Sleep reduced power usage by 6-fold, relative to the Active State, whereas Light Sleep reduced it by 3-fold.

\section{CONCLUSION}

In this study, we have evaluated the influence of DRX Light and Deep Sleep mode on power consumption for Voice and Web traffic in relation to the TTI size. In Voice traffic the use of Deep Sleep and Light Sleep both showed significant power reduction effect at a similar degree with a Static TTI size mode. It is approximated that TTI size of around $10 \mathrm{~ms}$ is the optimal for power efficiency with minimal adverse effects (i.e. delay-associated larger TTI) regardless of whether Deep Sleep or Light Sleep was applied. Our result of the adjustable TTI model also indicated that Deep Sleep and Light Sleep have similar power saving effect. There is a clear advantage of Light Sleep over Deep Sleep in that Light Sleep reduces the chance of delay in traffic because the sleep cycle of Light Sleep is shorter than that of Deep Sleep and therefore PDCCH can detect packet for scheduling in a timely manner [10]. We also evaluated the power consumption of Active, Deep Sleep and Light Sleep in Web traffic. The use of Deep Sleep and Light Sleep showed a significant power reduction, however unlike in the case of Voice traffic, Deep Sleep performed better in terms power saving. While Deep Sleep reached to the optimal power saving level as early as the frame size of
$8 \mathrm{~ms}$, Light Sleep reached to the optimal level at $14 \mathrm{~ms}$. Deep Sleep achieves power efficiency at smaller TTI frame size, which allows the UE to respond in a timely manner to assess whether there is data transmission to the UE. On the other hand, Light Sleep requires larger TTI frame size to reach acceptable level of power saving. Thus, combined use of Light Sleep and Deep Sleep may be the desirable method for maximum power efficiency with minimum delay.

\section{ACKNOWLEDGMENT}

Scott Fowler was partially supported by the Swedish Excellence Center at Linköping - Lund in information Technology (ELLIIT).

\section{REFERENCES}

[1] W. W. R. Forum, "[online]," www.wireless-world-research.org.

[2] R. Susitaival and M. Meyer, "Lte coverage improvement by tti bundling," in Vehicular Technology Conference, 2009. VTC Spring 2009. IEEE 69th, pp. $1-5$, april 2009.

[3] J. Han and H. Wang, "Principle and performance of tti bundling for voip in lte fdd mode," in Wireless Communications and Networking Conference, 2009. WCNC 2009. IEEE, pp. 1 -6, april 2009.

[4] M. Rinne and O. Tirkkonen, "Lte, the radio technology path towards 4g," Comput. Commun., vol. 33, pp. 1894-1906, October 2010.

[5] H. Wu and T. Haustein, "Energy and spectrum efficient transmission modes for the 3gpp-lte uplink," in Personal, Indoor and Mobile Radio Communications, 2007. PIMRC 2007. IEEE 18th International Symposium on, pp. 1-5, 2007.

[6] J.-H. Yeh, J.-C. Chen, and C.-C. Lee, "Comparative analysis of energysaving techniques in 3gpp and 3gpp2 systems," Vehicular Technology, IEEE Transactions on, vol. 58, no. 1, pp. $432-448,2009$.

[7] S.-R. Yang, S.-Y. Yan, and H.-N. Hung, "Modeling umts power saving with bursty packet data traffic," Mobile Computing, IEEE Transactions on, vol. 6, no. 12, pp. $1398-1409,2007$.

[8] S.-R. Yang and Y.-B. Lin, "Modeling umts discontinuous reception mechanism," Wireless Communications, IEEE Transactions on, vol. 4, no. 1 , pp. $312-319,2005$.

[9] G. T. 36.300, "Evolved universal terrestrial radio access (e-utra) and evolved universal terrestrial radio access network (e-utran)," www.3 gpp.org, vol. v10.2.0 (Release 9), December 2010.

[10] G. T. 36.321, "Evolved universal terrestrial radio access (e-utra) medium access control (mac) protocol specification," www.3gpp.org, vol. v10.0.0 (Release 10), December 2010.

[11] G. S. Kim, Y. H. Je, and S. Kim, "An adjustable power management for optimal power saving in lte terminal baseband modem," Consumer Electronics, IEEE Transactions on, vol. 55, no. 4, pp. 1847 -1853, 2009.

[12] H. Bo, T. Hui, C. Lan, and Z. Jianchi, "Drx-aware scheduling method for delay-sensitive traffic," Communications Letters, IEEE, vol. 14, no. 12, pp. $1113-1115,2010$.

[13] L. Zhou, H. Xu, H. Tian, Y. Gao, L. Du, and L. Chen, "Performance analysis of power saving mechanism with adjustable drx cycles in 3gpp lte," in Vehicular Technology Conference, 2008. VTC 2008-Fall. IEEE 68th, pp. $1-5,2008$.

[14] C. Bontu and E. Illidge, "Drx mechanism for power saving in lte," Communications Magazine, IEEE, vol. 47, no. 6, pp. 48 -55, 2009.

[15] W. Willinger, M. Taqqu, R. Sherman, and D. Wilson, "Self-similarity through high-variability: statistical analysis of ethernet lan traffic at the source level," Networking, IEEE/ACM Transactions on, vol. 5, pp. 71 -86, Feb. 1997.

[16] V. Paxson and S. Floyd, "Wide area traffic: the failure of poisson modeling," Networking, IEEE/ACM Transactions on, vol. 3, pp. 226 -244, June 1995.

[17] I.-T. R. P.59, "Telephone transmission quality objective measuring apparatus: Artificial conversational speech," International Telecommunication Union, March 1993.

[18] ETSI, "Universal mobile telecommunications system (umts);selection procedures for the choice of radio transmission technologies of the umts," Technical Report UMTS 30.03, version 3.2.0, April 1998.

[19] N. R2-071285, “3gpp ts 36.300," www.wireless-world-research.org, vol. 3GPP TSG RAN WG2 LTE, March 2007. 